\title{
Análisis de esfuerzos mecánicos generados en las articulaciones de rodilla y cadera durante la trayectoria total de la marcha humana
}

\author{
pags $82-97$
}

Grupo de Investigación: grupo de diseño avanzado

Línea de investigación: Biomecánica

Juan Caldas•, Sergio Beltrán»• \& Héctor Castro॰••

\section{RESUMEN}

En este trabajo se realizó un análisis por elementos finitos en la fase de apoyo de la marcha humana en las articulaciones de cadera y rodilla. Se encontró una aproximación de las magnitudes y distribución de esfuerzos en las articulaciones. Inicialmente se obtuvieron los modelos geométricos en 3D de las articulaciones de rodilla y cadera, luego se determinaron las fuerzas ejercidas por los músculos que afectan directamente las articulaciones con el programa OpenSim y se calcularon las fuerzas totales y momentos generados por las fuerzas durante la fase de apoyo del ciclo de marcha. Se establecieron las condiciones de frontera, puntos de aplicación de fuerzas, momentos y soportes fijos para el desarrollo de las simulaciones. Por último, se realizaron las simulaciones durante la fase de apoyo de la marcha humana en las articulaciones de rodilla y cadera donde se identificaron los posibles puntos de esfuerzo máximo de acuerdo a los resultados obtenidos con la aplicación del programa ANSYS.

Palabras clave: Blomecánica, Rodilla, Cadera, Esfuerzo, marcha humana, elementos finitos.

\section{ABSTRACT}

This article describes a finite element analysis of hip and knee joints in the stance phase of human gait. An approximation of magnitude and distribution of stresses was obtained for these joints. At first, geometric 3D models of hip and knee joint were obtained; the forces produced by the main muscles that affect directly hip and knee joints were registered with OpenSim, in order to determine the total moments and forces generated in the joints through the human gait. Boundary conditions, points of application of forces, moments and fixed supports were defined and simulations were developed during the stance phase. The approximate points of maximum stresses during the stance phase were determined with the results obtained with the application of ANSYS.

Keywords: Biomechanics, Knee, Hip, Stress, human gait, finite elements.

\footnotetext{
- Estudiante coinvestigador del programa de Ingeniería mecánica. Fundación Universidad de América. juan.caldas@estudiantes. uamerica.edu.co

-. Estudiante coinvestigador del programa de Ingeniería mecánica de la Fundación Universidad de América Sergio.beltran@ estudiantes.uamerica.edu.co

..* Ingeniero mecatrónico magister en ingeniería biomecánica, docente investigador de la Fundación Universidad de Amércia. Hector.castro@profesores.uamerica.edu.co
} 
LINNEA DE INVESTIGACIÓN: BIOMECÁNICA

\section{INTRODUCCIÓN}

La articulación de rodilla y cadera permiten el movimiento de la pierna en actividades cotidianas como saltar, caminar y correr, soportando a la vez el peso corporal. Tanto la cadera como la rodilla, son articulaciones expuestas a esfuerzos mecánicos que pueden ser provocados por exceso de fuerzas externas o sobrecargas aplicadas en el miembro inferior durante la trayectoria total de la marcha humana.

La trayectoria total de la marcha se encuentra comprendida por la fase de apoyo y la fase de balanceo. La fase de apoyo se divide en cinco intervalos de posición: El contacto inicial, respuesta a la carga, apoyo medio, apoyo final y pre-balanceo (Cortes \& Lagos, 2011). Investigaciones relacionadas con análisis de esfuerzos en rodilla y cadera, han demostrado que se presentan altos valores de esfuerzos mecánicos en el miembro inferior y durante los intervalos de posición correspondientes a la fase de apoyo de la marcha. Mientras que en la fase de balanceo, los esfuerzos son menores y constantes (Guo, Zhang, \& Chen, 2009), (Lotz, Cheal, \& Hayes, 1995).

Teniendo en cuenta lo anterior, en este artículo se realiza un análisis de esfuerzos mecánicos presentes en la articulación de rodilla y cadera, de una persona sana. En donde se describen los valores máximos de esfuerzo presentes en los intervalos de posición de la fase de apoyo de la marcha. Para esto se llevó a cabo un estudio biomecánico de la articulación de rodilla y cadera sin patologías, mediante un software especializado. Al inicio de esta investigación se describe la anatomía de las articulaciones de rodilla y cadera, especificando los principales músculos que participan en el movimiento de flexión y extensión del miembro inferior. Luego se detallan los modelos geométricos en 3D de rodilla y cadera, seguido de las condiciones de carga y de frontera calculadas sobre las articulaciones durante la fase de apoyo de la marcha. Por último se describen las simulaciones que evidencian los esfuerzos críticos en las articulaciones.

\section{MATERIALES Y MÉTODOS}

De acuerdo con (Guo, Zhang, \& Chen, 2009) y (Godest, Beaugonin, Haug, Taylor, \& Gregson, 2002), las fuerzas que se generan en la articulación de la rodilla durante la fase de balanceo son constantes y se pueden asumir como despreciables, por lo anterior y de acuerdo a las siguientes gráficas, el presente trabajo se realizó teniendo en cuenta la fase de apoyo de la marcha humana, es decir, del $0 \%$ al $60 \%$ de la trayectoria total de la marcha humana. De la misma manera se asumieron las fuerzas de la articulación de la cadera, haciendo referencia a (Von Eisenhart, Adam, Steinlechner, Müller-Gerbl, \& Eckstain, 1999)

Figura 1: Fuerzas en las articulaciones de Rodilla y Cadera

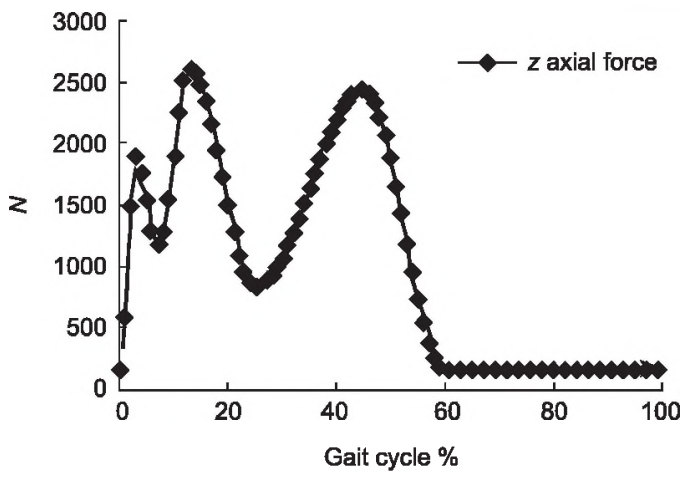

a) Fuerzas en la articulación de la rodilla

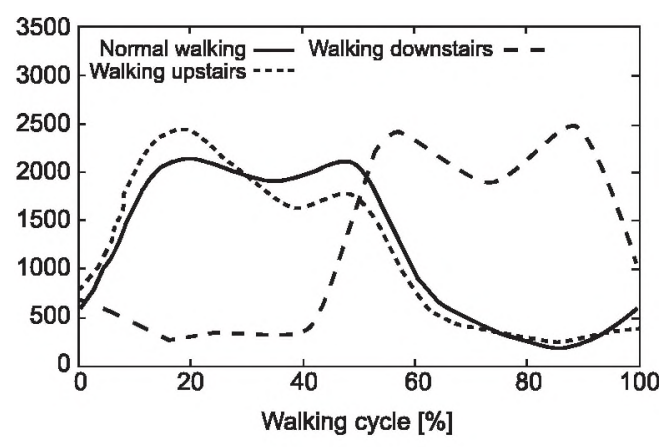

b) Fuerzas en la articulación de la cadera

Fuente: los autores. 


\subsection{Modelos geométricos}

Los modelos de rodilla y cadera (Figura 2) utilizados en la investigación se obtuvieron de Simtk, sitio en internet que se especializa en el estudio biomecánico del cuerpo humano (SimTK, 2015). Los modelos adquiridos se desarrollaron con base en una tomografía computarizada de la extremidad inferior derecha de una persona sana, con masa corporal de $75 \mathrm{Kg}$ y estatura de $1,70 \mathrm{~m}$.
Para el desarrollo de la simulaciones durante la fase de apoyo de la marcha se deben tener en cuenta los músculos que actúan sobre las articulaciones, de acuerdo con (Chaoitow, Walker DeLany, Lowe, \& Vaugh, 2006), en la tabla 1 se muestran los músculos utilizados para determinar las fuerzas que se generan en las articulaciones.

Figura 2: Modelo geométricos de las articulaciones de Rodilla y Cadera

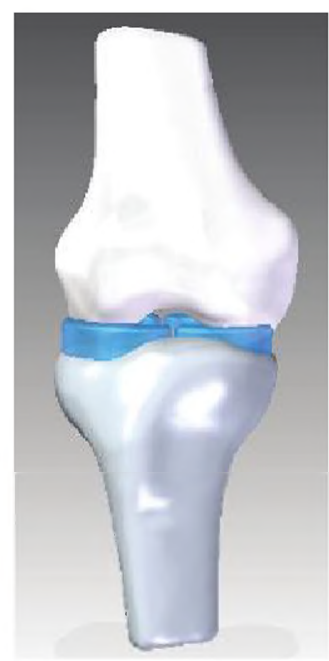

a) Modelo geométrico de la articulación de la rodilla

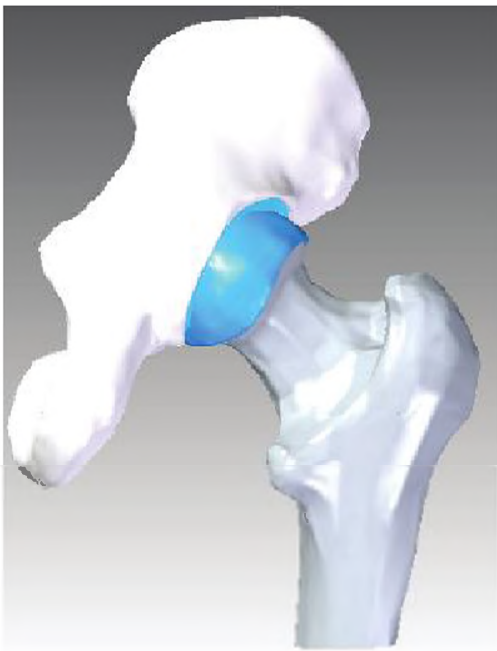

b) Modelo geométrico de la articulación de la cadera

Fuente: los autores.

Tabla 1: Músculos principales que actúan durante la fase de apoyo de la marcha humana en la articulación de rodilla y cadera

\begin{tabular}{|c|c|c|c|}
\hline \multicolumn{4}{|c|}{ Músculos } \\
\hline & Rodilla & & Cadera \\
\hline \multirow{4}{*}{ Cuádriceps } & Recto femoral & \multirow{3}{*}{$\begin{array}{l}\text { Isquiotibiales } \\
\text { combinados }\end{array}$} & Bíceps femoral (cabeza larga) \\
\hline & Vasto Lateral & & Semimembranoso \\
\hline & \begin{tabular}{|l|} 
Vasto Medial \\
\end{tabular} & & Semitendinoso \\
\hline & Vasto intermedio & \multirow{7}{*}{ Otros } & Psoas Mayor \\
\hline \multirow{3}{*}{ Isquiotibiales } & Bíceps femoral cabeza larga & & Glúteo Mayor \\
\hline & Semimembranoso & & Iliaco \\
\hline & Semitendinoso & & \begin{tabular}{|l|} 
Tensor de la Fascia Lata \\
\end{tabular} \\
\hline \multirow{2}{*}{ Gastrocnemios } & Gastrocnemio medial & & Aductor Mayor \\
\hline & Gastrocnemio lateral & & Aductor medio \\
\hline Otro & Bíceps femoral cabeza corta & & Sartorio \\
\hline
\end{tabular}

Fuente: los autores. 
LIINEA DE INVESTIGACIÓN: BIOMECÁNICA

\subsection{Fuerzas y momentos en las articulaciones de rodilla y cadera}

Una vez los músculos que actúan sobre las articulaciones fueron establecidos, se encontraron las fuerzas y momentos ejercidos por los músculos sobre la rodilla y cadera durante la fase de apoyo de la marcha humana por medio del software OpenSim (Delp, y otros, 2007). Este software permite obtener la fuerza que ejerce cada uno de los músculos que se relacionan directamente con la rodilla y cadera en cada uno de los instantes de la fase de apoyo. De la misma manera se puede establecer el sentido de la fuerza dependiendo de la dirección de la curva, es decir, cuando el musculo se extiende, se encuentra en tracción y en dirección positiva en el eje Y. Cuando se recoge se encuentra en compresión y en dirección negativa en el eje Y.

Con la aplicación de todas las fuerzas que ejerce cada uno de los músculos en la rodilla y cadera se realizó una sumatoria de fuerzas en cada porcentaje de la fase de apoyo y se encontró la fuerza total que ejercen los músculos en la articulación de la rodilla y cadera durante esta fase.

En las figuras 3 y 4 se observan las componentes vectoriales de las fuerzas de cada músculo en cada porcentaje de la marcha en la fase de apoyo. En el desarrollo de las simulaciones se ingresaron en ANSYS las componentes en î y j de las fuerzas calculadas, motivo por el que se presentan de esta manera.

Figura 3. Fuerza total en la rodilla contra porcentaje de la fase de apoyo de la marcha

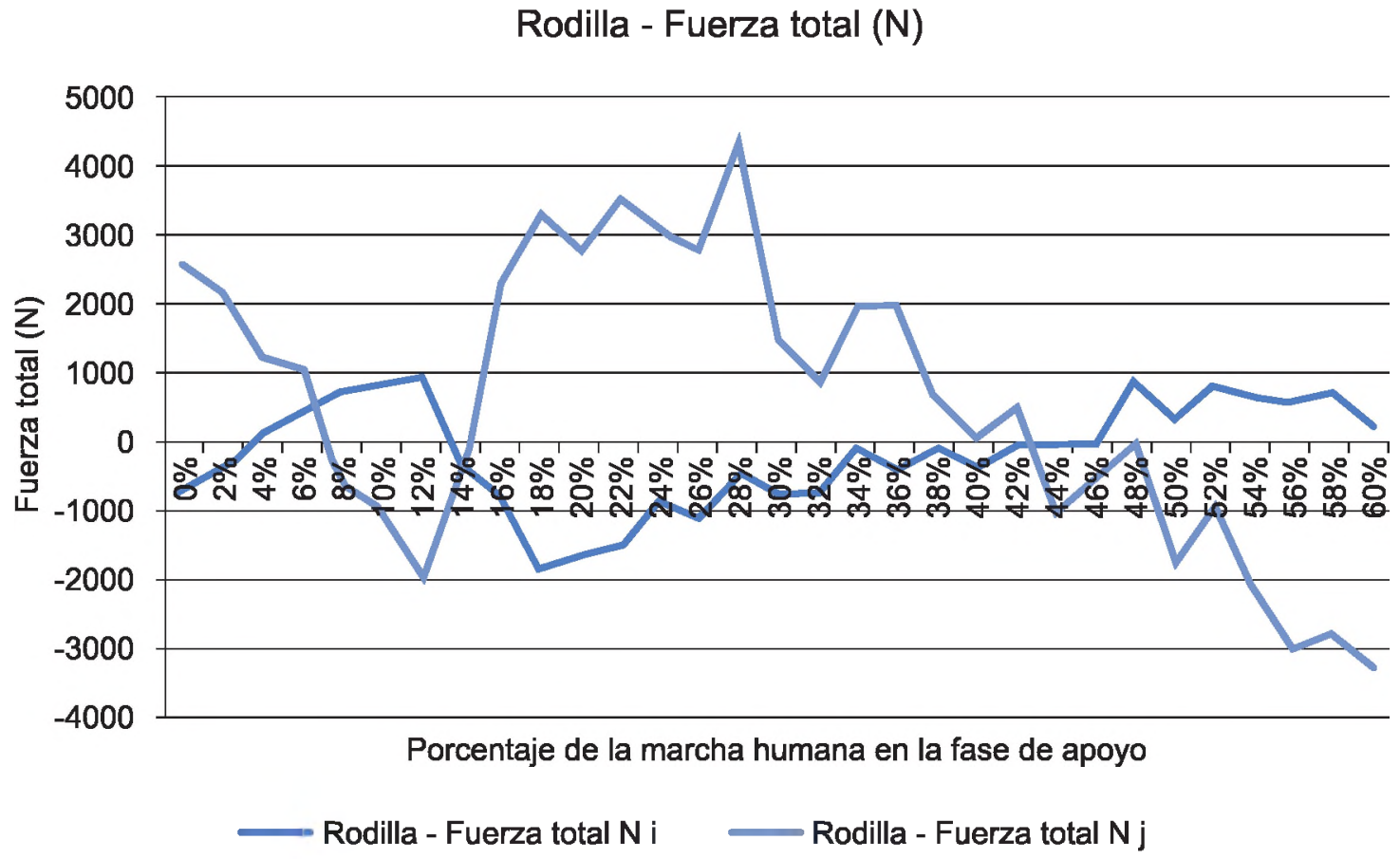

Fuente: los autores.

Mediante el producto cruz entre las fuerzas encontradas y un punto en común definido como punto de corte, se encontraron los momentos generados en las articulaciones de rodilla y cadera, estos se muestran en las figuras 5 y 6 . 
Figura 4. Fuerza total en la cadera contra porcentaje de la fase de apoyo de la marcha

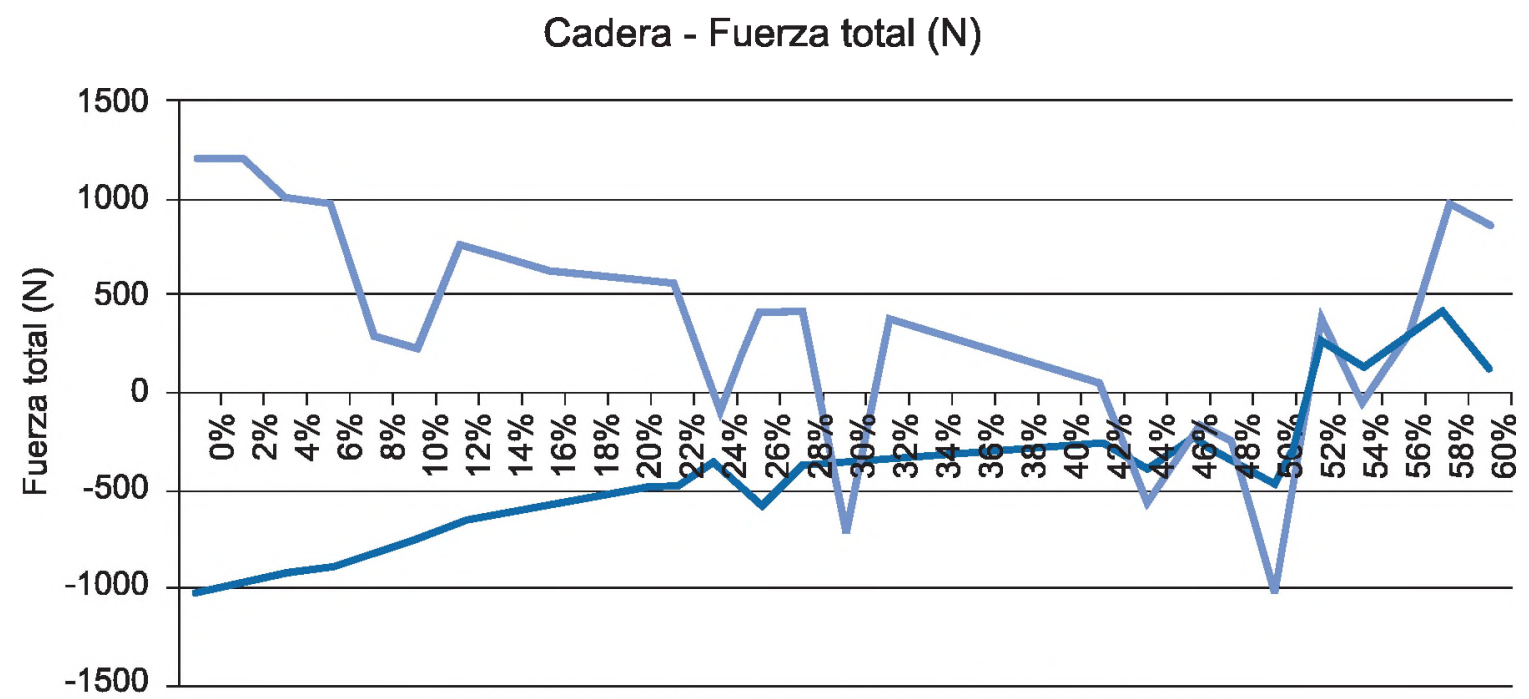

Porcentaje de la marcha humana en la fase de apoyo

Rodilla - Fuerza total N i Rodilla - Fuerza total N j

Fuente: los autores.

Figura 5. Momentos en la rodilla contra porcentaje de la fase de apoyo de la marcha

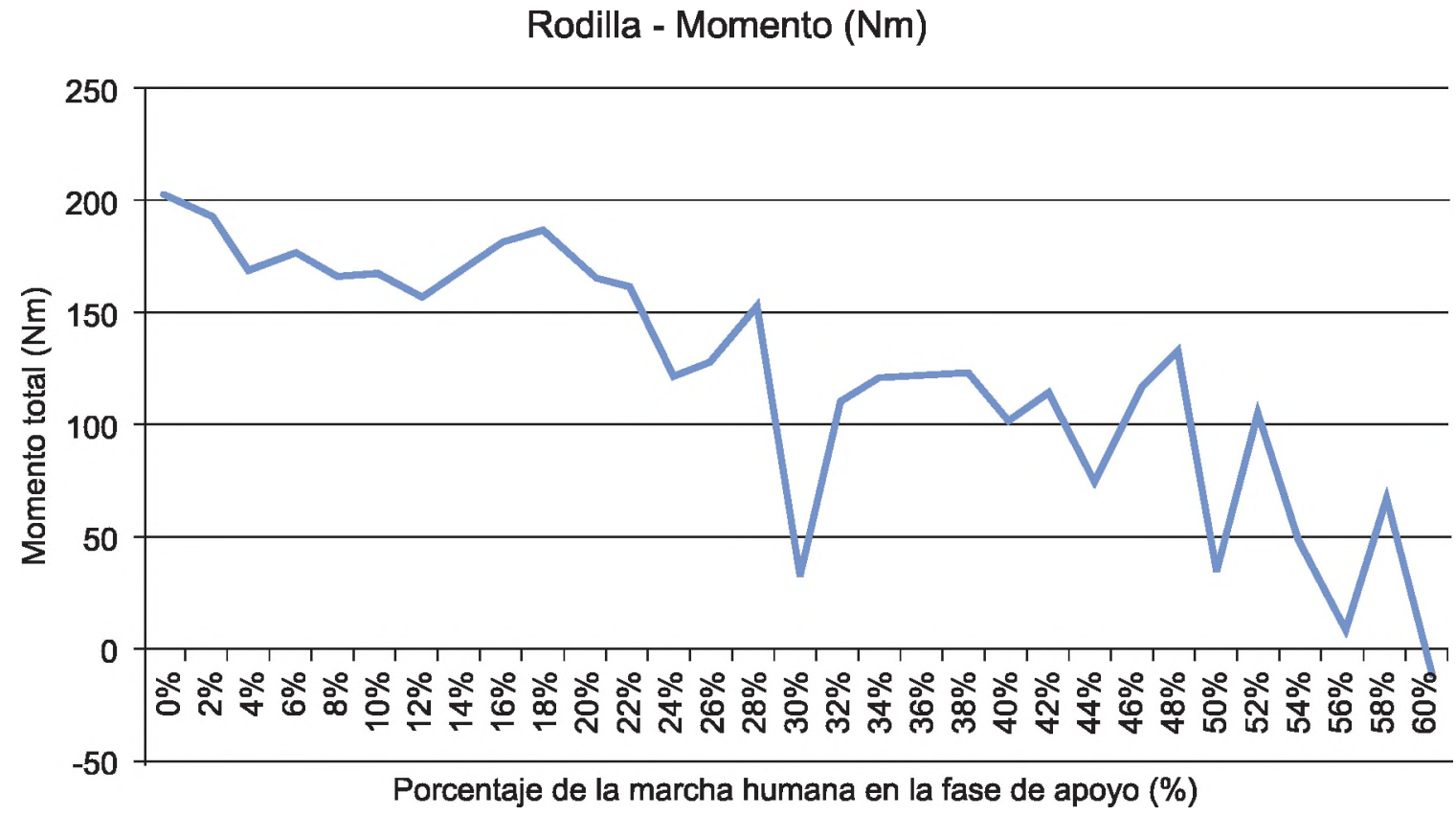

Fuente: los autores. 
LÍNEA DE INVESTIGACIÓN: BIOMECÁNICA

Figura 6. Momentos en la cadera contra porcentaje de la fase de apoyo de la marcha.

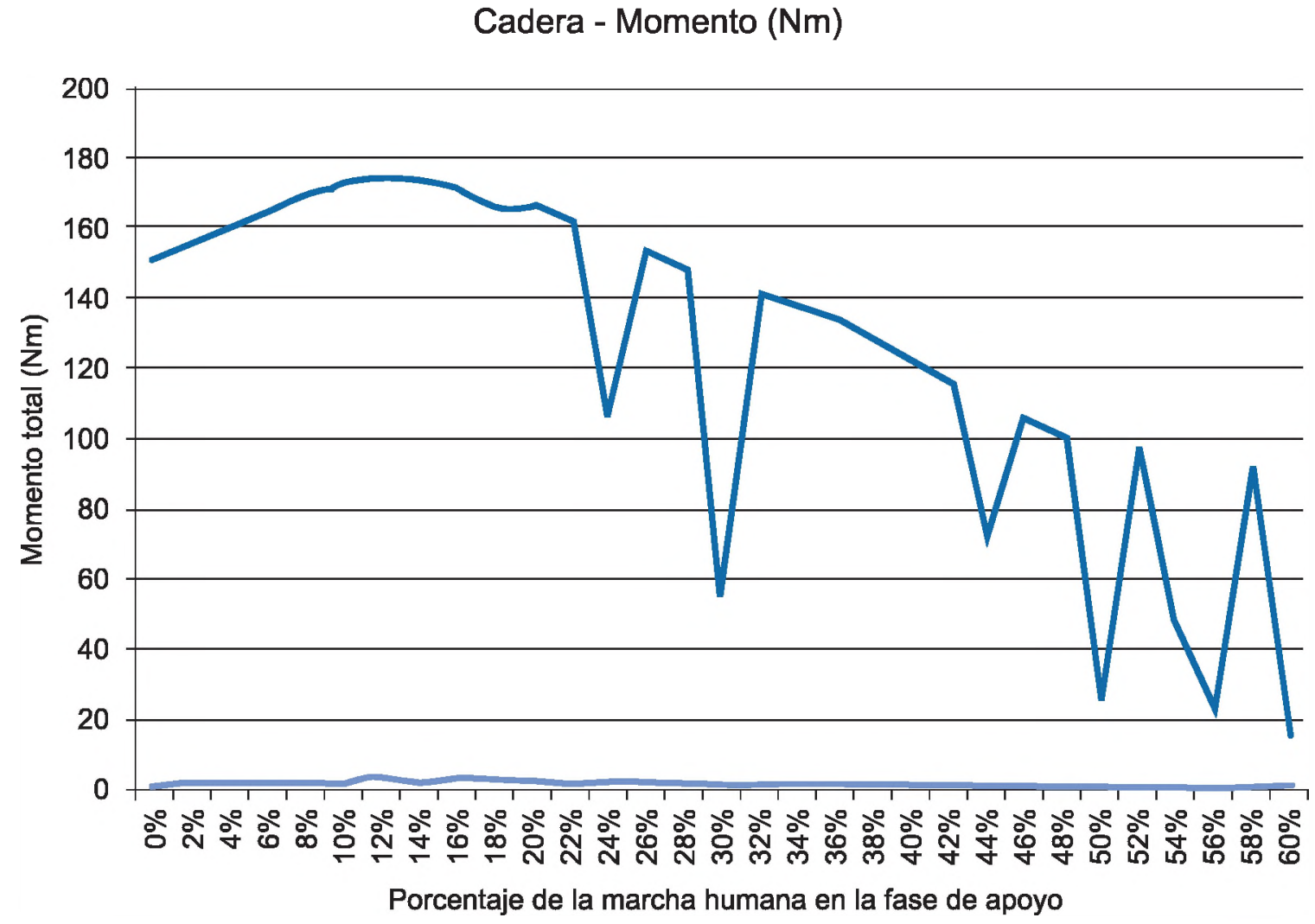

Fuente: los autores.

\subsection{Condiciones de frontera}

Con respecto a las características del hueso y de las componentes de las articulaciones, se manejaron materiales isotrópicos lineales, haciendo relación a diferentes autores como (Donahue, Hull, Rashid, \& Jacobs, 2002) y (Harris, y otros, 2012). Adicionalmente, se definieron las características de cada material (Tabla 2) consultando artículos de distintos autores.

Para la simulación se ingresaron los modelos geométricos en 3D de cada porcentaje de la fase de apoyo de la marcha, se definieron las propiedades de los materiales y se incluyeron las condiciones de frontera (Ver figura 7 y 8 ).

Tabla 2. Propiedades Isotrópicas de los materiales de los componentes de la rodilla

\begin{tabular}{|l|c|c|l|}
\hline \multicolumn{1}{|c|}{ Componente } & Módulo Elástico (MPa) & Coefieiente de Poisson & \multicolumn{2}{c|}{ Autor } \\
\hline Hueso Cortical & 17000 & 0,3 & (Sunghyen \& Mistugu, 2013) \\
\hline Meniscos & 59 & 0,49 & $\begin{array}{l}\text { (Peña, Calvo, Martínez, Palanca, \& } \\
\text { Doblaré, 2005) }\end{array}$ \\
\hline Hueso Trabecular & 1500 & 0,33 & (Voo, Armand, \& kleinberger, 2004) \\
\hline Cartílagos & 15 & 0,3 & (Park, Kim, Kim, \& Oh, 2009) \\
\hline
\end{tabular}

Fuente: los autores. 
Figura 7. Cargas y restricciones en el modelo de la rodilla en ANSYS
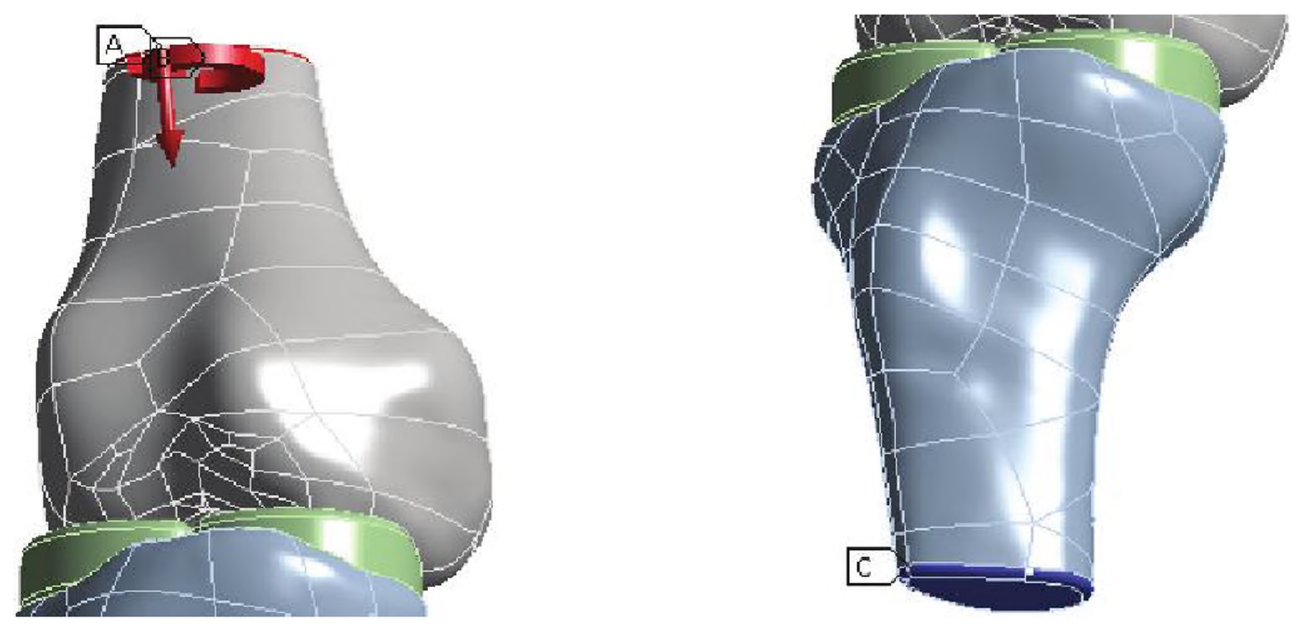

Figura 8. Cargas y restricciones en el modelo de la cadera en ANSYS

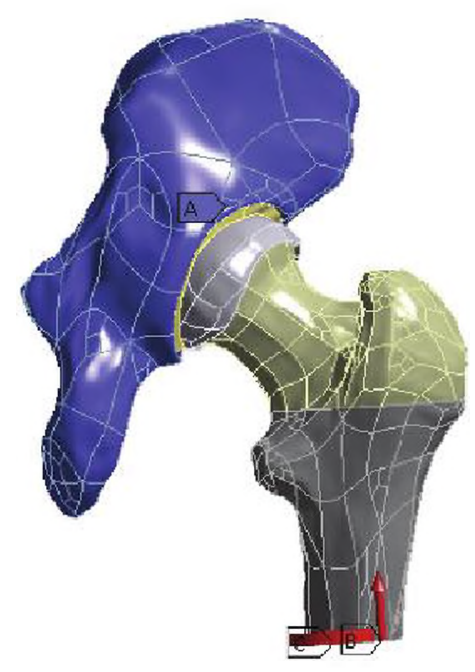

Fuente: los autores.

Se modificó la geometría en 3D de la rodilla y cadera para hacer las simulaciones de acuerdo a su posición en cada porcentaje de la marcha y del ángulo que forman el fémur y la tibia para cada instante. Sin embargo al hacer las simulaciones modificando los ángulos de la articulación, se encontró que los esfuerzos máximos superaban el esfuerzo último de los materiales ya que se generaban concentradores de esfuerzo por la penetración de la tibia y el fémur en los meniscos. Por lo tanto, se decidió no modificar los ángulos del fémur con respecto a la tibia y aplicar las fuerzas en el modelo geométrico 3D de la fase inicial de la marcha. Se realizó un análisis geométrico para su traslado encontrando las nuevas componentes $X$ y $Y$ de las fuerzas.

Se realizó un análisis de sensibilidad de malla para identificar el tamaño adecuado de elemento para aplicar a las características del modelo. Ver tabla 3 y 4 . 
Tabla 3. Número de elementos promedio en el enmallado de la articulación de la Rodilla

\begin{tabular}{|c|c|c|}
\hline $\begin{array}{l}\text { Número de } \\
\text { en el enmall }\end{array}$ & os pr & $10 \mathrm{r}$ \\
\hline Meniscos & Fémur & Thbis \\
\hline 5983 & 7375 & 5737 \\
\hline
\end{tabular}

Fuente: los autores.

Tabla 4. Número de elementos promedio en el enmallado de la articulación de la Cadera

Número de elementos promedio de elementos

en el enmallado de la Articulación de la Cadera

\begin{tabular}{c|c|c|}
$\begin{array}{c}\text { Cartilago } \\
\text { femoral }\end{array}$ & Fémur & $\begin{array}{c}\text { Cartilago } \\
\text { acetabular }\end{array}$ \\
\hline 8368 & 9446 & 8368 \\
\hline
\end{tabular}

\section{RESULTADOS}

Se realizaron las simulaciones en los porcentajes de la fase se apoyó de la marcha humana, en las siguientes figuras subsiguientes se muestra el resultado de las simulaciones en el contacto inicial, respuesta a la carga, apoyo medio, Apoyo final y pre-balanceo respectivamente. También se observan los resultados de esfuerzo durante toda la fase de apoyo de la marcha humana para las dos articulaciones. Con estas graficas se evidencian los valores máximos de esfuerzo encontrados en las simulaciones y se establecen de manera aproximada los puntos críticos de esfuerzo durante la marcha humana.

Fuente: los autores.

\subsection{Articulación de la Rodilla}

Figura 9. Simulación y resultados del fémur en los instantes de contacto inicial, respuesta a la carga, apoyo medio, Apoyo final y pre-balanceo.

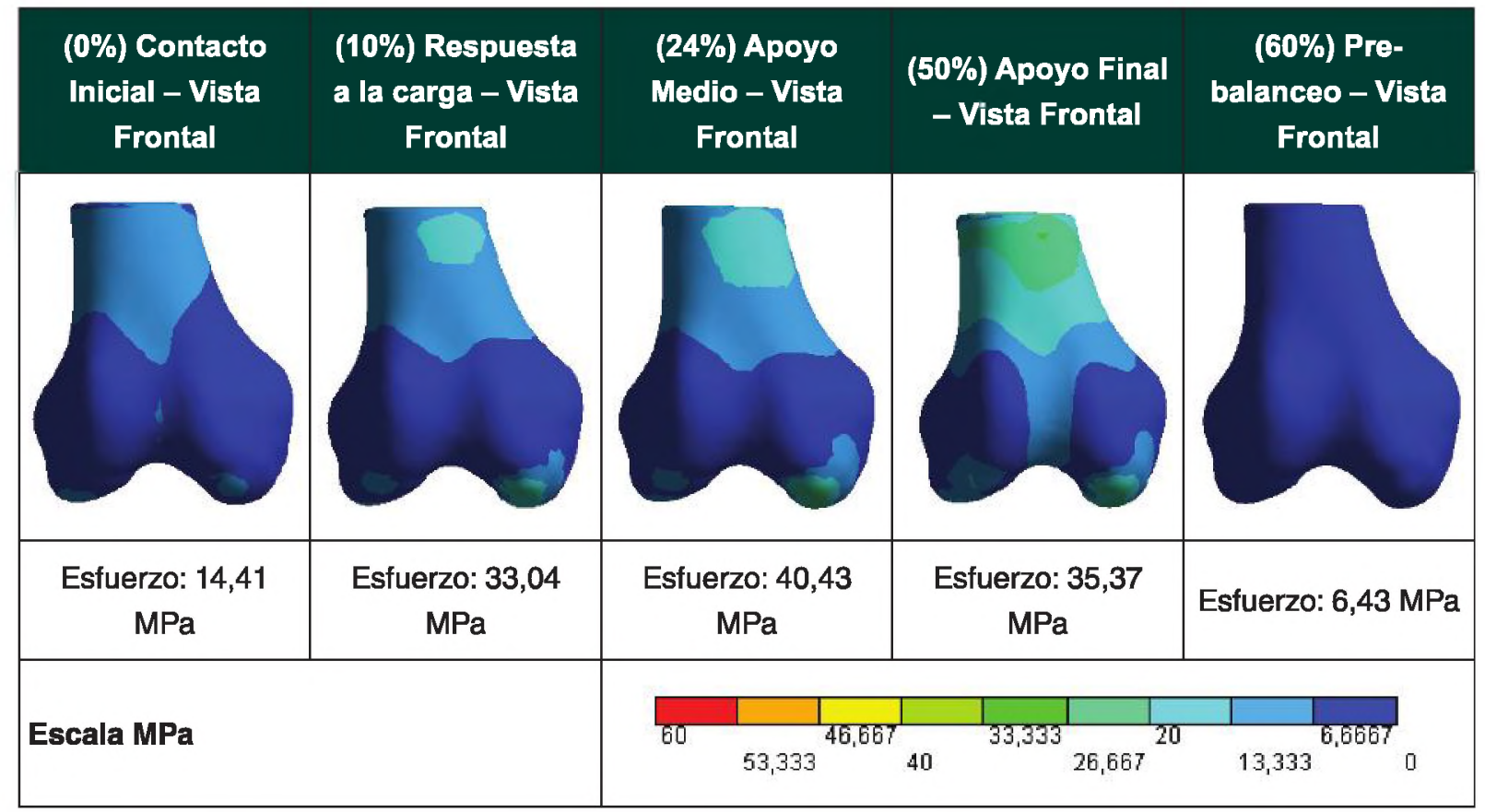

Fuente: los autores. 
Figura 10. Simulación y resultados de los meniscos en los instantes de contacto inicial, respuesta a la carga, apoyo medio, Apoyo final y pre-balanceo.

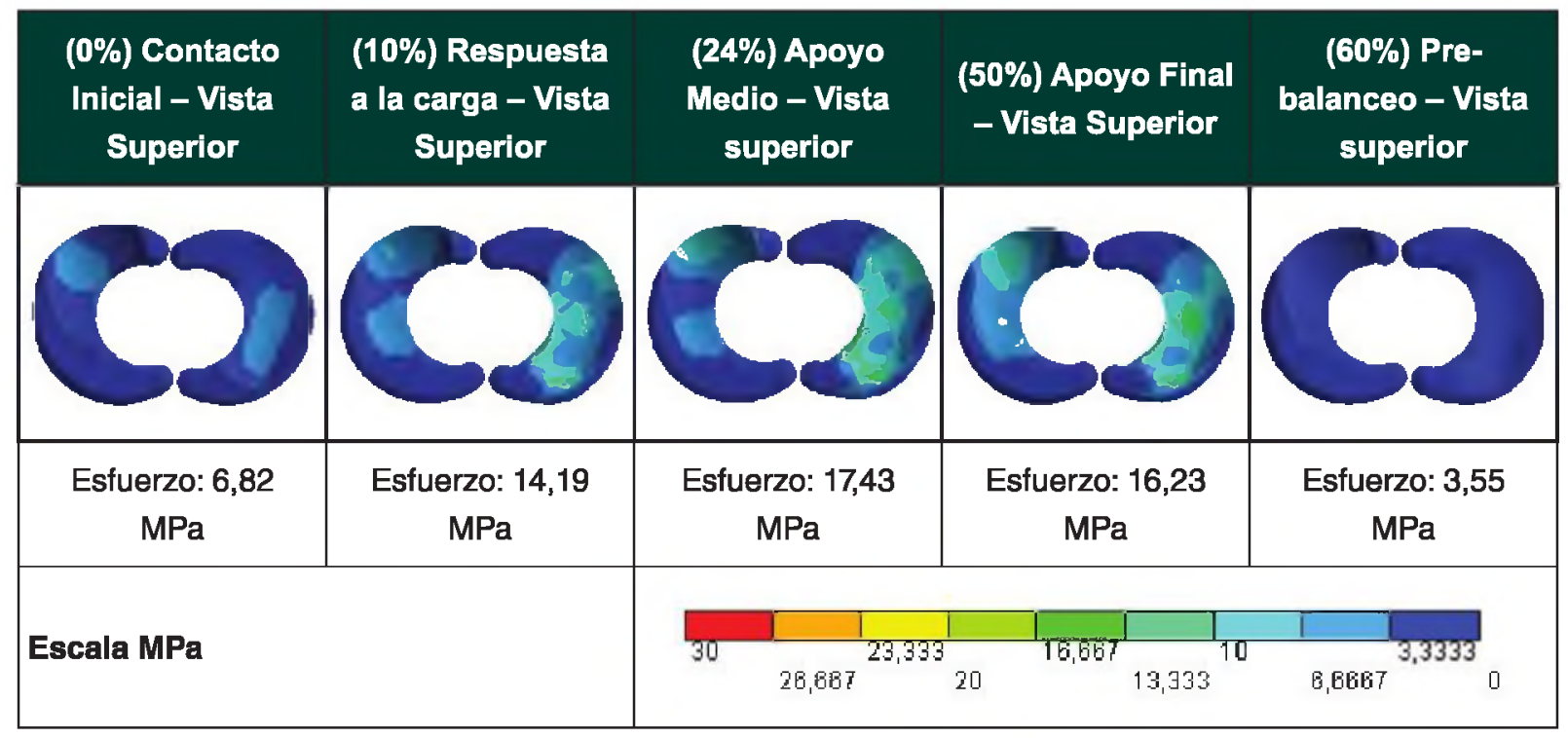

Fuente: los autores.

Figura 11. Simulación y resultados de la tibia en los instantes de contacto inicial, respuesta a la carga, apoyo medio, Apoyo final y pre-balanceo.

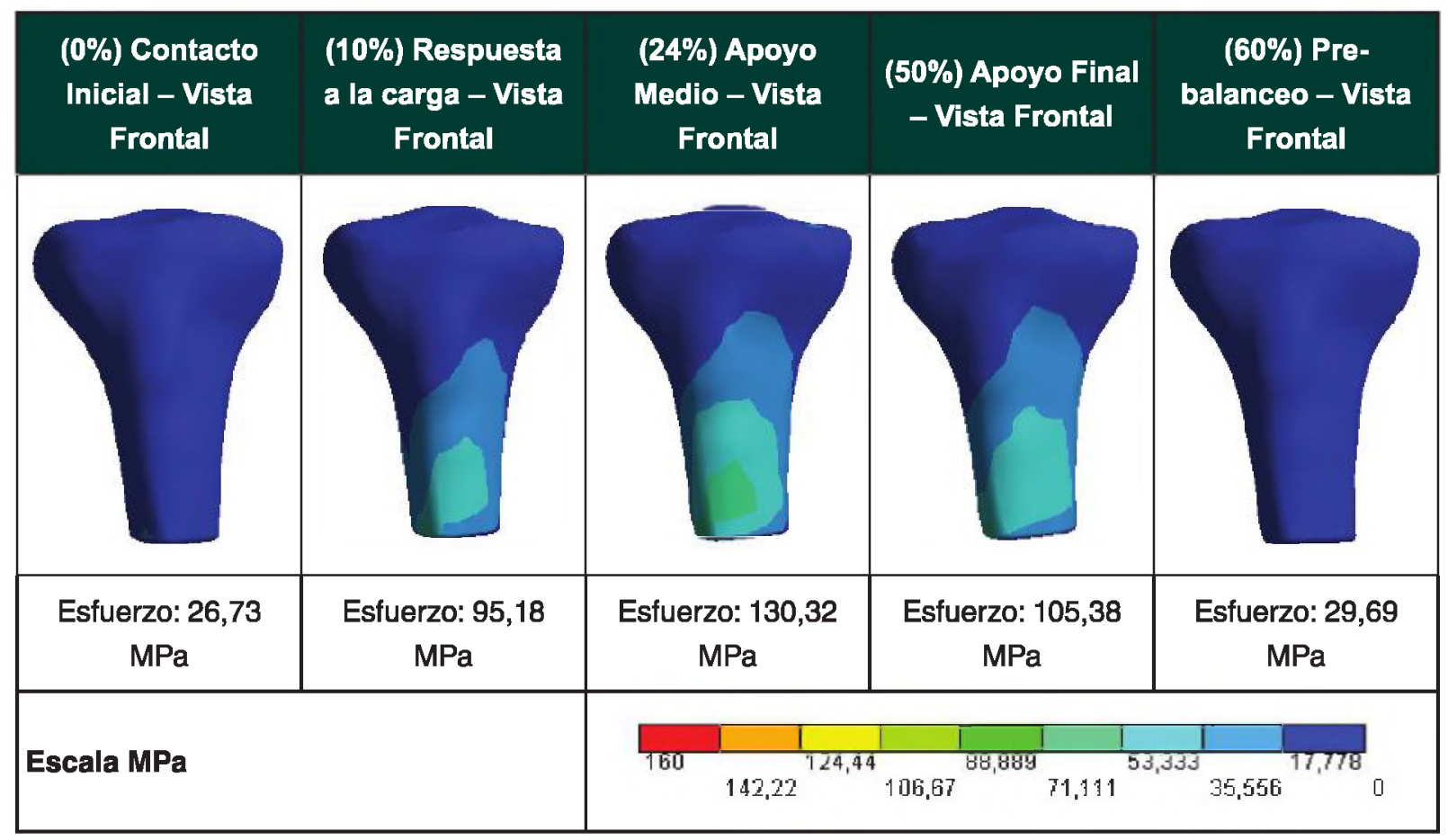

Fuente: los autores. 
LÍNEA DE INVESTIGACIÓN: BIOMECÁNICA

\subsection{Articulación de la Cadera}

Figura 12. Simulación y resultados del fémur en los instantes de contacto inicial, respuesta a la carga, apoyo medio, apoyo final y pre-balanceo.

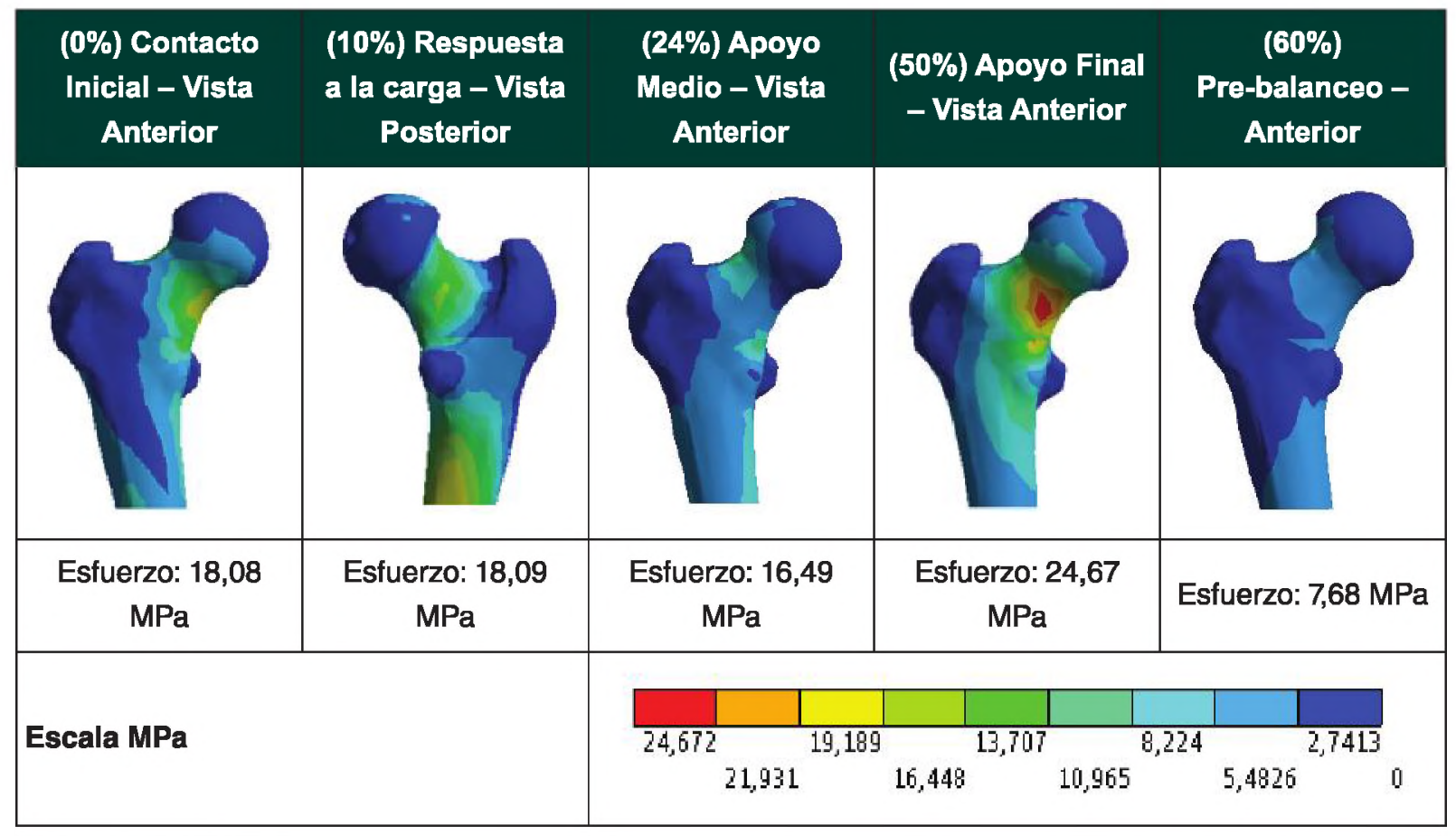

Fuente: los autores.

Figura 13. Simulación y resultados del cartílago acetabular en los instantes de contacto inicial, respuesta a la carga, apoyo medio, apoyo final y pre-balanceo

\begin{tabular}{|l|l|l|l|l|l|}
\hline $\begin{array}{c}\text { (0\%) Contacto } \\
\text { Inicial - Vista } \\
\text { Superior }\end{array}$ & $\begin{array}{c}\text { (10\%) Respuesta } \\
\text { a la carga - Vista } \\
\text { Superior }\end{array}$ & $\begin{array}{c}\text { (24\%) Apoyo } \\
\text { Medio - Vista } \\
\text { Superior }\end{array}$ & $\begin{array}{c}\text { (50\%) Apoyo Final } \\
\text { - Vista Superior }\end{array}$ & $\begin{array}{c}\text { (60\%) Pre- } \\
\text { balanceo- } \\
\text { Superior }\end{array}$ \\
\hline & & & & \\
\hline
\end{tabular}

Fuente: los autores. 
Figura 14. Simulación y resultados del cartílago femoral en los instantes de contacto inicial, respuesta a la carga, apoyo medio, Apoyo final y pre-balanceo

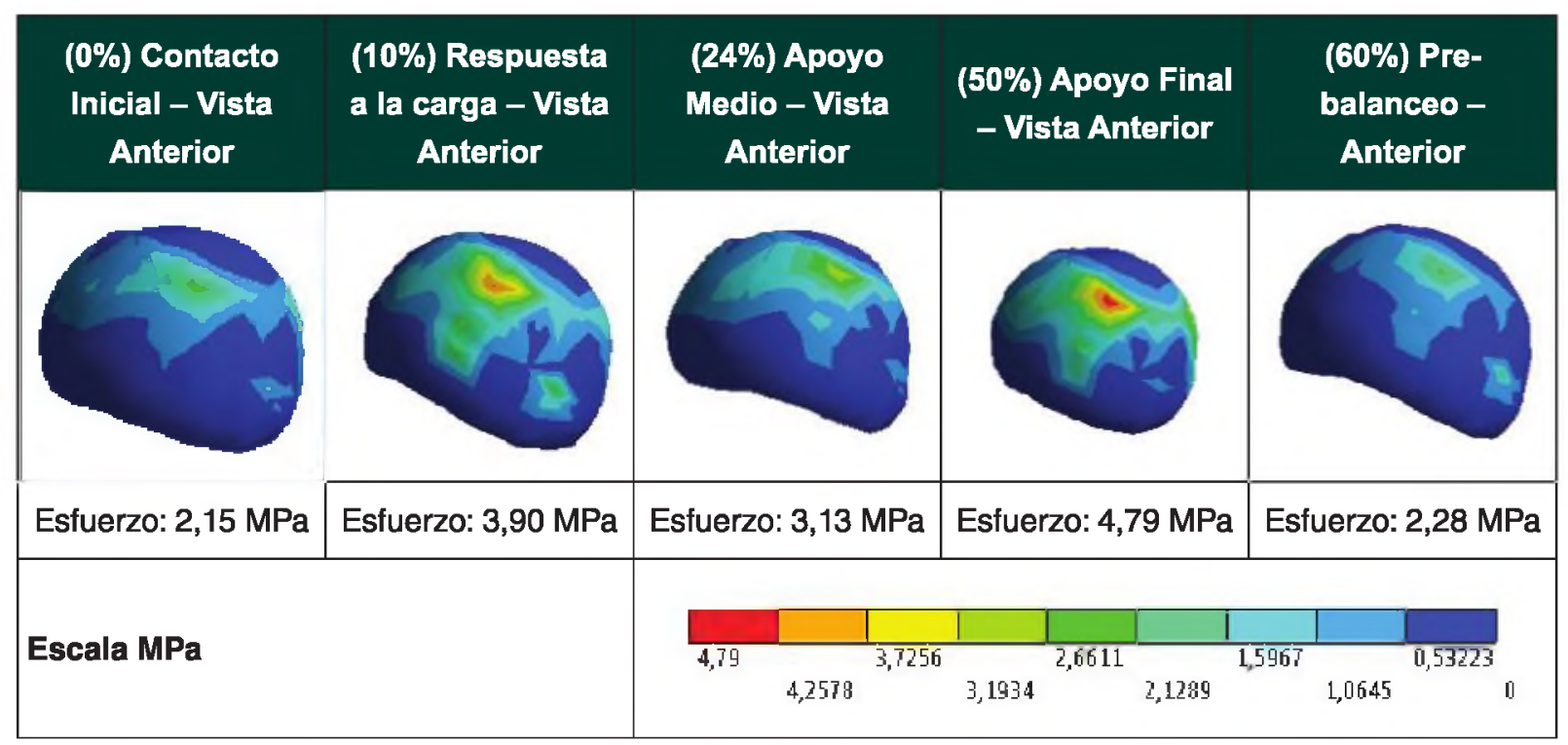

Fuente: los autores.

\section{DISCUSIÓN}

\subsection{Articulación de la Rodilla}

Figura 15. Esfuerzos presentes en fémur durante la fase de apoyo de la marcha humana

Esfuerzos presentes en el Fémur (MPa) - Articulación de la rodilla

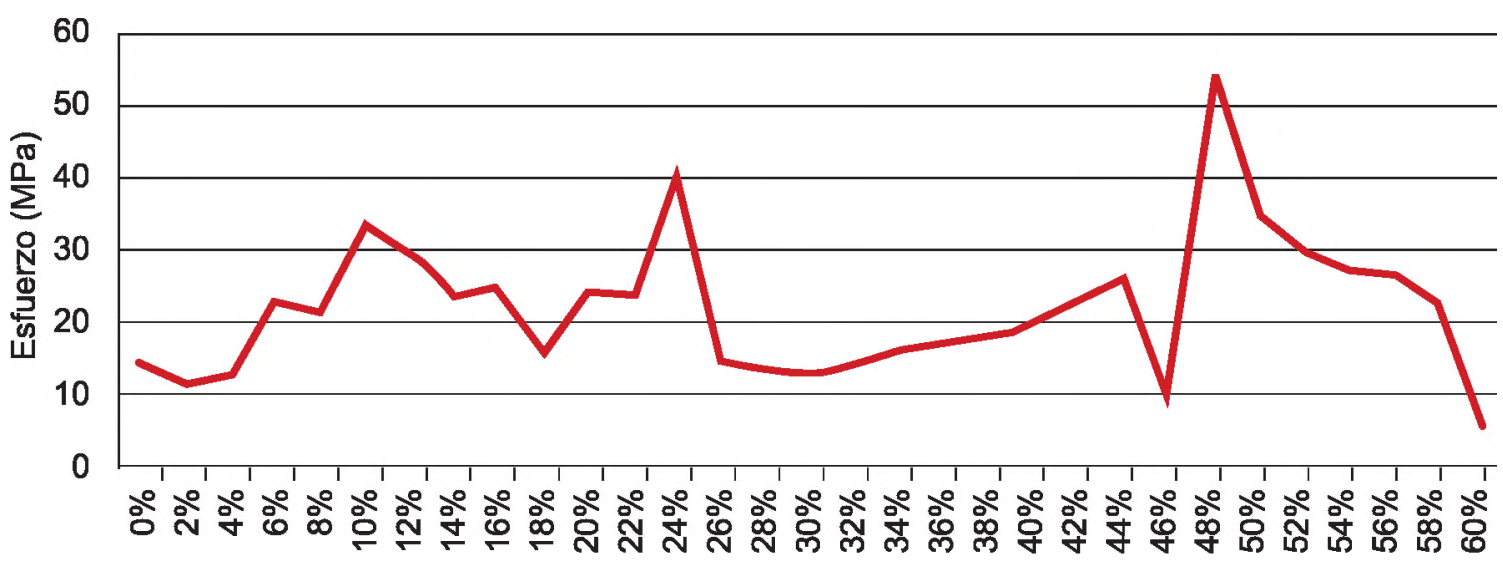

Porcentaje de la marcha humana (\%)

Fuente: los autores. 
LÍNEA DE INVESTIGACIÓN: BIOMECÁNICA

Figura 16. Esfuerzos presentes en los meniscos durante la fase de apoyo de la marcha humana Esfuerzos presentes en los meniscos (MPa) - Articulación de la rodilla

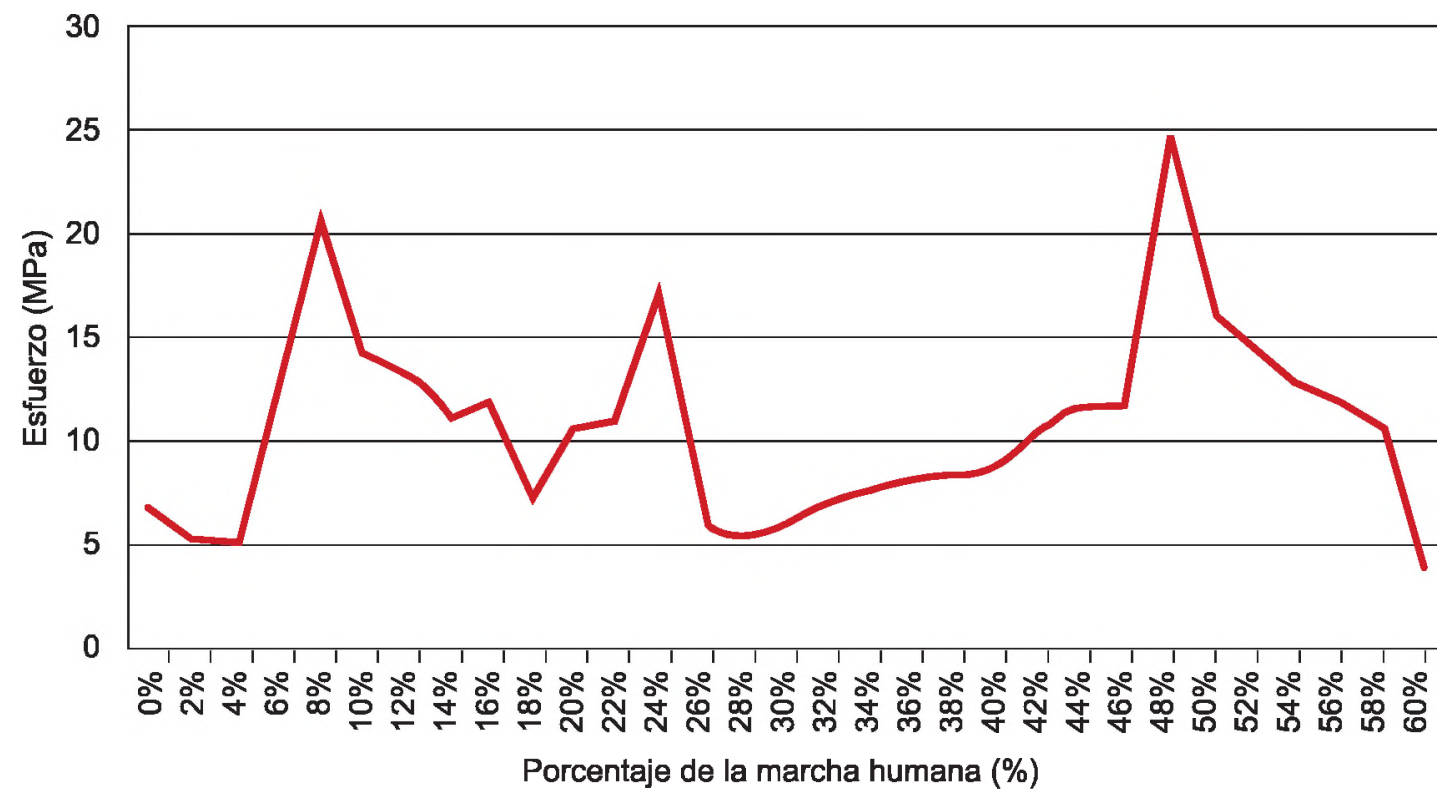

Fuente: los autores.

igura 17. Esfuerzos presentes en la tibia durante la fase de apoyo de la marcha humana Esfuerzos presentes en la tibia (MPa) - Articulación de la rodilla

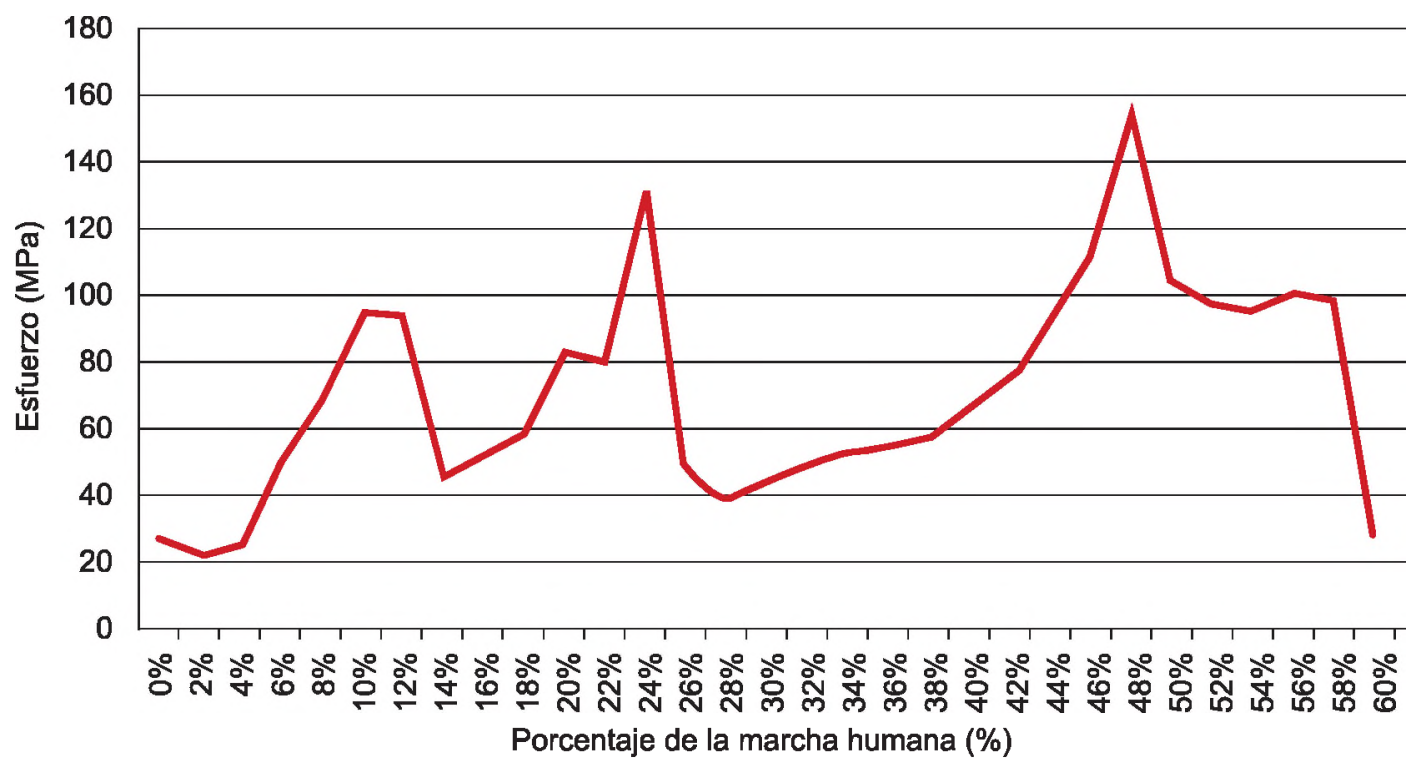

Fuente: los autores. 
Los resultados obtenidos por medio de las simulaciones en ANSYS para identificar los puntos de esfuerzo máximo indican que para la articulación de la rodilla se presenta un esfuerzo de contacto máximo en los meniscos de 24,88 MPa. Este valor con la literatura fue comparado con resultados de otros autores; se hace referencia a (Guo, Zhang, \& Chen, 2009), donde se encontró un valor máximo de esfuerzo de $21 \mathrm{MPa}$ para el contacto en los meniscos. Lo anterior indica que los resultados obtenidos se acercan a trabajos anteriores. Adicionalmente en el trabajo realizado por (Godest, Beaugonin, Haug, Taylor, \& Gregson, 2002) se encontró que el esfuerzo máximo de contacto en los meniscos fue de $22 \mathrm{MPa}$.

Se obtuvo un esfuerzo de $17,43 \mathrm{MPa}$ en la fase de apoyo medio o en el $24 \%$ de la trayec- toria total de la marcha. De acuerdo con (Guo, Zhang, \& Chen, 2009), en esta fase de la marcha se presenta un esfuerzo de aproximadamente $15 \mathrm{MPa}$. Adicionalmente, para el $50 \%$ y $60 \%$ de la marcha humana, en este trabajo se obtuvo un esfuerzo en los meniscos de 16,23 MPa y 3,55 MPa respectivamente, valores cercanos a los obtenidos por (Guo, Zhang, \& Chen, 2009) que fueron de $16 \mathrm{MPa}$ en el $50 \%$ y 10 MPa en el $60 \%$ de la marcha humana.

Para la articulación de la rodilla, en el $8 \%$, $24 \%$ y $48 \%$ de la marcha humana se presentan valores de esfuerzo altos que se deben en cuenta para el desarrollo de futuros estudios o fabricación de prótesis de bajo costo.

\subsection{Articulación de la Cadera}

Figura 18. Esfuerzos presentes en el fémur durante la fase de apoyo de la marcha humana

$$
\text { Esfuerzos presentes en el fémur (MPa) - Articulación de la cadera }
$$

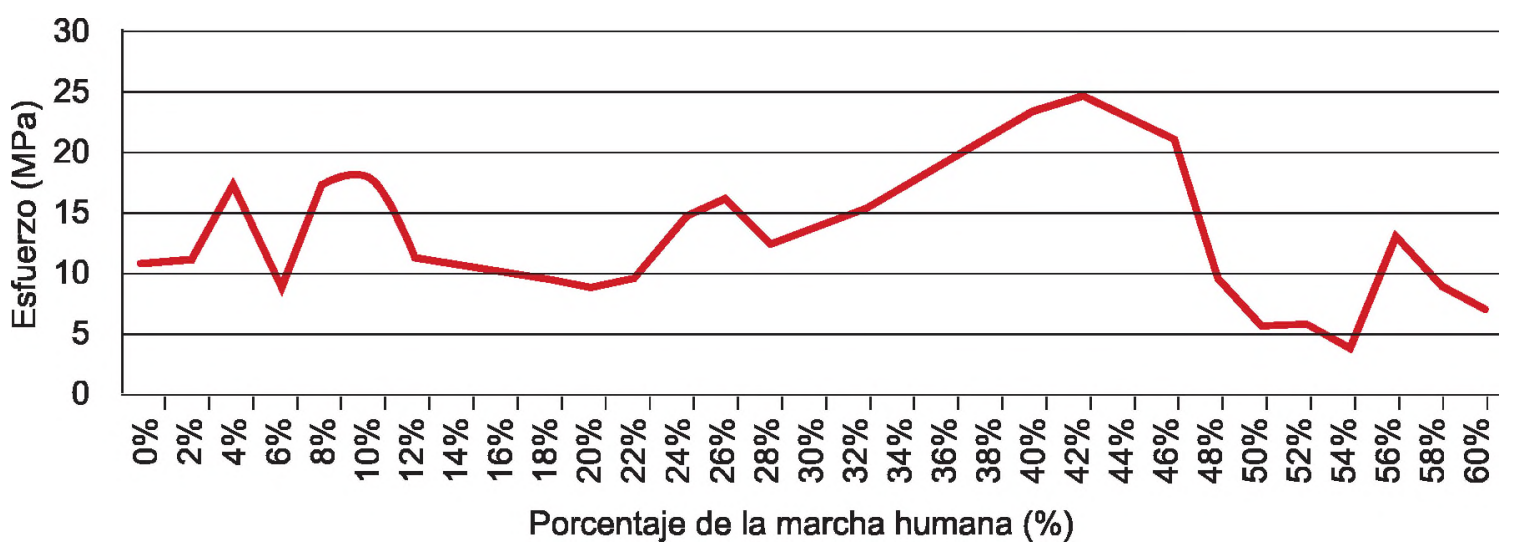

Fuente: los autores.

Se encontró que durante la fase de apoyo de la marcha, los esfuerzos máximos presentes en el fémur, cartílago acetabular y femoral de la articulación de la cadera, ocurren en los instantes del contacto inicial e inicios del apoyo de la marcha. Según (Krebs, Elbaum, Riley, Hodge, \& Mann, 1991), los picos máximos de esfuerzo se presentan en el cartílago femoral y acetabular con magnitud de 3,78 y $3,47 \mathrm{MPa}$ respectivamente. Otros autores como (Abt, Altekruse, \& Brinckmann, 1981) describen picos de esfuerzos de 2,4 y 3,2 MPa durante la fase de contacto inicial de la marcha. En esta investigación, se presentaron picos de esfuerzos de 4,79 MPa en el cartílago femoral, 5,07 $\mathrm{MPa}$ en el cartílago acetabular y $25 \mathrm{MPa}$ en el fémur, durante el contacto inicial y apoyo medio de la marcha. 
LIINEA DE INVESTIGACIÓN: BIOMECÁNICA

Figura 19. Esfuerzos presentes en el cartílago acetabular durante la fase de apoyo de la marcha humana

Esfuerzos presentes en el cartílago Acetabular (MPa) -

Articulación de la cadera

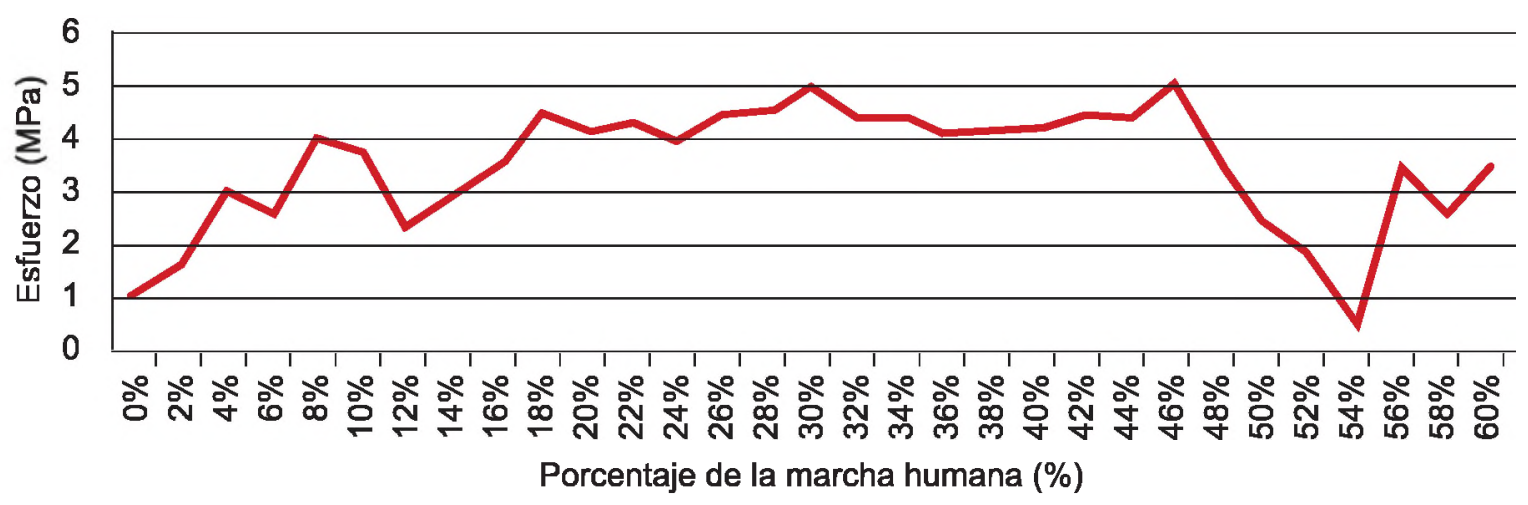

Fuente: los autores.

Figura 20. Esfuerzos presentes en el cartílago femoral durante la fase de apoyo de la marcha humana

Esfuerzos presentes en el cartílago Femoral (MPa) Articulación de la cadera

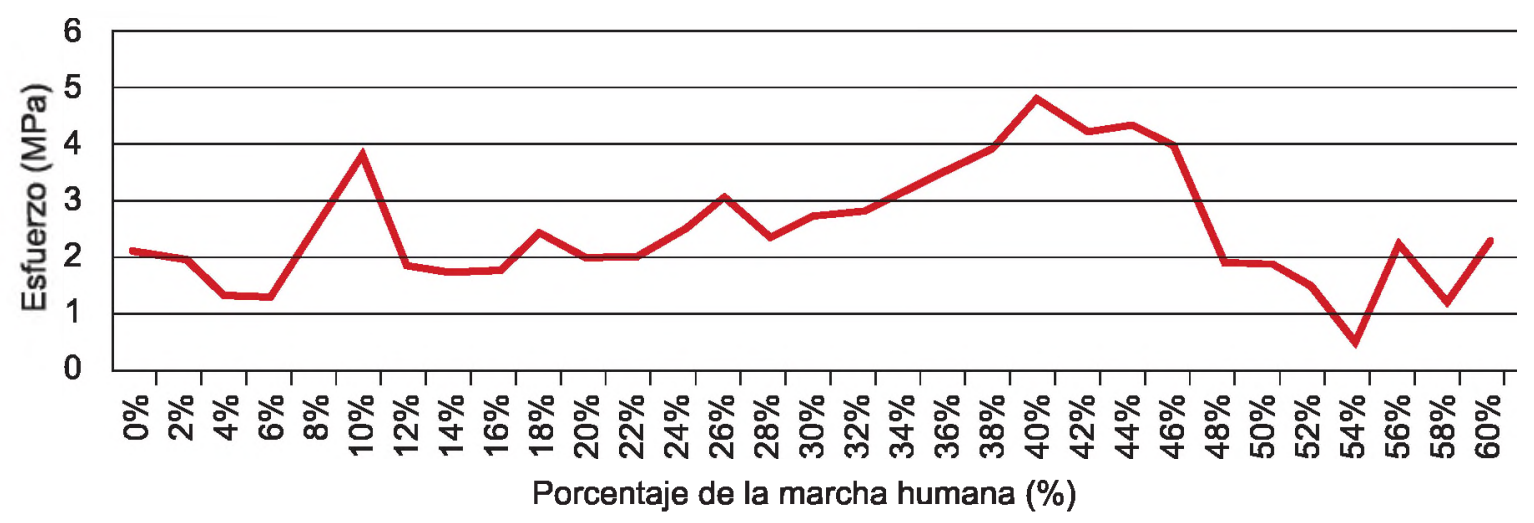

Fuente: los autores.

En los análisis de esfuerzos realizados en la cadera, se evidenció que en el fémur proximal, específicamente en el cuello femoral, se concentraron esfuerzos máximos del orden 20 a 25 MPa. Valores de esfuerzo cercanos a los presentados por (Lotz, Cheal, \& Hayes, 1995), los cuales estuvieron en el orden de $16 \mathrm{MPa}$ en el fémur proximal sano. Los esfuerzos máximos se concentran principalmente en el cuello femoral debido al cambio significativo en la geometría proximal del fémur.

\section{CONCLUSIONES}

En este trabajo se realizó un análisis por el método de elementos finitos de la articulación 
de la rodilla y cadera con el programa ANSYS durante la fase de apoyo de la marcha humana. Para esto se obtuvieron los modelos geométricos en 3D de las articulaciones de rodilla y cadera, luego se obtuvieron las fuerzas ejercidas por los músculos que actúan directamente sobre cada articulación con el software especializado OpenSim y se calcularon las fuerzas totales y los momentos generados por las fuerzas. Se establecieron las condiciones de frontera y se procedieron a hacer las simulaciones durante la fase de apoyo de la marcha humana en las articulaciones de rodilla y cadera. Finalmente, se establecieron los puntos de esfuerzo máximo.

El objetivo principal de este trabajo era definir si se presentan esfuerzos mayores en diferentes fases de la marcha humana teniendo en cuenta los resultados de los trabajos realizados en la Universidad de América y diferentes trabajos disponibles de consulta. Al hacer la comparación se concluye que se presenten esfuerzos altos diferentes a los estudios realizados anteriormente. Este artículo podría ser considerado en futuras investigaciones ya que muestra magnitudes de esfuerzos diferentes y mayores a los estudios realizados anteriormente en la Universidad de América.

Con el desarrollo de los análisis de sensibilidad de malla se obtuvieron mejores resultados, el tamaño y número de elementos de malla utilizados en las condiciones de frontera dieron resultados adecuados al compararlos con diferentes trabajos de otros autores. Trabajos desa- rrollados previamente anteriormente sin análisis de convergencia de malla muestran unos valores de esfuerzos en los meniscos con una diferencia del orden de $120 \mathrm{MPa}$ en la fase de apoyo final. De esta manera se evidencia la importancia de realizar o verificar la convergencia del enmallado.

Investigaciones realizadas en la Universidad de América (Figueroa, 2014), describen que los esfuerzos máximos presentes en el fémur, cartílago acetabular y femoral, sucedieron durante el $45 \%$ de la fase de apoyo de la marcha, es decir, durante la fase final. Al comparar esos resultados con esta investigación, se evidencia que los esfuerzos máximos del cartílago acetabular se presentaron en el $46 \%$ de la marcha. En el cartílago femoral durante el $40 \%$ y fémur en el $42 \%$. Porcentajes que pertenecen a la fase final de la marcha humana.

En conclusión, en este artíclo se describen los análisis de esfuerzos mecánicos que se presentan en los cartílagos y huesos de la articulación de rodilla y cadera. El cálculo de los esfuerzos presentes durante la fase de apoyo de la marcha humana se debe tener en cuenta en futuras investigaciones para el desarrollo de prótesis y análisis de la marcha, y así evitar fracturas que pueden ser causadas por sobre cargas aplicadas en las articulaciones de rodilla y cadera. Para obtener resultados óptimos en los análisis de esfuerzos se debe tener en cuenta la implementación de una malla fina, lo cual requiere una alta capacidad en el procesador del computador.

\section{REFERENCIAS}

Abt, B., Altekruse, M., \& Brinckmann, P. (1981). "Stress on the articular surface of the hip joint in persons with idiopathic coxarthrosis and healthy adults (author's transl)". Zeitschrift für orthopädie un lhre Grenzgebiete, 119(4), 382-386.
Chaoitow , N. D., Walker DeLany, J., Lowe, J., \& Vaugh, B. (2006). "Aplicación clínica de las técnicas neuromusculares II: Extremidades inferiores". Barcelona, España: Editorial Paidotribo.

Cortes, L., \& Lagos, M. J. (20 de 06 de 2011). Loco- moción Humana. Obtenido de http://analisisdelamarcha. blogspot.com.co/

Delp, S. L., Anderson, F. C., Arnold, A. S., Loan, P., Habib, A., John, C. T., ... Thelen, D. G. (2007). "OpenSim: opensource software to create and analyze dynamic simulations of 
movement". IEEE transactions on bio-medical engineering, 54(11), 1940-1950.

Donahue, T. L., Hull, M. L., Rashid, M. M., \& Jacobs, C. R. (2002). "A finite element model of the human knee joint for the study of tibio-femoral contact". Journal of Biomechanical Engineering, 124(3), 273-280.

Figueroa, J. F. (2014). “Determinación del comportamiento mecánico de las patologías de la articulación coxofemoral de mayor incidencia en Colombia ". Trabajo de Grado. Bogotá, Colombia: Fundación Universidad de América.

Godest , A. C., Beaugonin, M., Haug, E., Taylor, M., \& Gregson, P. J. (2002). "Simulation of a knee joint replacement during a gait cycle using explicit finite element analysis". Journal of Biomechanics, 32(2), 267275.

Gómez Ibarra, R. E. (2013). "Modelado del comportamiento mecánico de la rodilla humana con las patologías de mayor incidencia en Colombia". Trabajo de grado. Bogotá, Colombia: Fundación Universidad de América.
Guo, Y., Zhang, X., \& Chen, W. (2009). "Three-Dimensional finite element simulation of total knee joint gait cycle". Acta Mechanica Solida Sinica, 22, 347-351.

Harris, M. D., Anderson, A. E., Henak, C. R., Ellis, B. J., Peters, C. L., \& Weiss, J. A. (2012). "Finite element prediction of cartilage contact stresses in normal human hips". Journal of Orthopaedic Research, 30(7), 1133-1139.

Krebs, E. D., Elbaum, L., Riley, P. O., Hodge, W. A., \& Mann, R. W. (1991). "Exercise and gait effects on in vivo hip contact pressures". Physical Therapy, 71(4), 301-309.

Lotz, J. C., Cheal, E. J., \& Hayes, W. C. (1995). "Stress distributions within the proximal femur during gait and falls: implications for osteoporotic fracture". Oseoporosis international Journal, 5(4), 252-261.

Park, W. M., Kim, Y. H., Kim, K., \& Oh, Y. T. (2009). "Non-destructive Biomechanical enalysis to evaluate surgical planning for hip joint diseases". International Journal of Precision Engineering and Manufacturing, 10(3), 12-131.
Peña, E., Calvo, B., Martínez, M. A., Palanca, D., \& Doblaré, M. (2005). "Finite element analysis of the effect of meniscal tears and meniscectomies on human knee biomechanics". Clinical Biomechanics, 20(5), 498-507.

Sunghyen, H., \& Mistugu, T. (2013). "Biomechanical Effect of Implantation of Chitosan/ MWNTs Reinforced Scaffold into Damaged Femur". Journal of Novel Carbon Resource Sciences, 7, 1-6.

VonEisenhart, R., Adam, C., Steinlechner, M., Müller-Gerbl, M., \& Eckstain, F. (1999). "Quantitative determination of joint incongruity and pressure distribution during simulated gait and cartilage thickness in the human hip joint". Journal of orthopaedic Research, 17(4), 532-539.

Voo, L., Armand, M., \& kleinberger, M. (2004). "Stress Fracture Risk Analysis of the Human Femur Based". john Hopkins APL Technical Digest, 25(3), 223-230. 